\title{
Characters of a Group of Bacillus Phages
}

\author{
By ELINOR W. MEYNELL \\ Departments of Bacteriology, London School of Hygiene and Tropical \\ Medicine and the Wright-Fleming Institute of Microbiology, \\ St Mary's Hospital, Praed Street, London, W. 2
}

(Received 10 August 1961)

\begin{abstract}
SUMMARY
The general characters are given of a group of phages related to phage $\beta$, a temperate phage carried by Bacillus cereus strain W (McCloy, 1951, $1953,1958)$. One phage, $\beta^{\prime}$, has been isolated only once but may be a mutant of phage $\beta$ since it, and its clear plaque variant, $\gamma^{\prime}$, have many properties in common with the other phages.
\end{abstract}

\section{INTRODUCTION}

Several phages can be isolated which attack Bacillus anthracis; some have already been discussed (McCloy, 1951, 1958). A description of their general characters is now given in view of the growing interest in the genetics of the genus Bacillus.

\section{METHODS}

The organisms and methods used were mostly described by McCloy (1951, 1958).

Phage-resistant variants. These were obtained by exposing Bacillus anthracis strain Davis to the non-lysogenizing phages $\alpha, \gamma$ or $\gamma^{\prime}$. All were similar in their cultural characters, which differed markedly from those of the original phagesensitive strain. Instead of forming flat grey rough colonies which were easily emulsified, and giving almost uniformly turbid broth cultures consisting of short chains of bacilli, they produced glistening white domed colonies which were stringy and tenacious, and grew in broth as a deposit of longer tangled chains, leaving a clear supernatant fluid. Complete phage resistance was invariably reached through a stage of partial resistance in which the bacteria produced thin rhizoid colonies on plates spread with phage, and colonies similar to those of the completely resistant variant when phage was absent. Repeated subculture of the partially resistant bacteria in the presence of phage led to the isolation of completely resistant variants which produced white-domed colonies in the presence of phage.

Adsorption to typical and atypical strains of Bacillus anthracis. In ordinary nutrient media incubated in air, where the mucoid capsule is not formed (Sterne, 1937), typical strains of Bacillus anthracis grow as long chains of bacilli, producing rough colonies on agar and a tangled deposit in broth. Smoother colony variants (Nungester, 1929), which grow in shorter chains, were isolated by incubating cultures for long periods (3-7 days) in large volumes $(100 \mathrm{ml}$.) of broth to which $0.025 \%$ (w/v) $\mathrm{CaCl}_{2}$ had sometimes been added (Bordet \& Renaux, 1930; Renaux, 1952).

In comparing the rates of adsorption to different strains, the bacterial sus- 
pensions were adjusted as nea ly as possible to the same optical density by eye. Although the degree of dispersion of suspensions of the different strains varied so much that their turbidities could not be accurately compared, the difference between adsorption to typical and to smoother-colony strains was so great that strictly comparable conditions were not ne:cessary to demonstrate it. Mixtures of $10^{6}$ to $10^{7}$ phage particles $/ \mathrm{ml}$. and about $5 \times 10^{8}$ bacilli $/ \mathrm{ml}$. were held at $37^{\circ}$, and the degree of absorption was measured eithe by assaying residual free phage after the bacteria had been removed by centrifugstion, or by assaying the number of infected bacteria after free phage had been inact vated by antiserum.

Suspensions of phage-infected spores. Nutrient agar plates were inoculated with bacteria and sufficient phage to ;ive semiconfluent lysis, and incubated for 3-4 days to allow sporulation to occur. The bacterial growth was then taken up in distilled water and incubated for several days to allow completion of sporulation and autolysis of vegetative bacteria (Hardwick \& Foster, 1952). The spores were then washed three times in distilled water ar d the final suspension was passed through double thicknesses of filter paper to remove large clumps. Filtration was repeated until no large clumps were seen microscopically, and the number of small aggregates was less than $2 \%$ of the number of single spores. Satisfactory suspensions were heated at 75-80 ${ }^{\circ}$ for $10-15 \mathrm{~min}$; t this treat ment reduced the viable count of uninfected spore suspensions about five-fold, and it was observed with phage-infected suspensions that the combined counts of colonies and plaques were always very considerably less than the total spore count estimated microscopically. The ratio of colonies : plaques in the different suspensions was between 4 and $8: 1$.

\section{RESULTS}

The phages used included: $(a) \beta$, a temperate phage carried by Bacillus cereus, strain $W ;(b) \alpha$, a virulent mutant of phage $\beta$ which can form plaques on strain $W$; (c) $\gamma$, another non-lysogenizing mutant of phage $\beta$ which, unlike $\alpha$, could not form plaques on strain $W$ or other strains lysogenized by phage $\beta ;(d) \beta^{\prime}$, a temperate phage which can form plaques on B. anthracis, strain Davis, lysogenized by phage $\beta$; (e) $\gamma^{\prime}$, a non-lysogenizing mutant of phage $\beta^{\prime}$ which did not form plaques on Davis $\left(\beta^{\prime}\right)$.

The source of phages $\alpha, \beta$ and $\gamma$ were given in McCloy $(1951,1958)$. Phage $\beta^{\prime}$ was isolated only once from turbid plaques formed on strain Davis $(\beta)$ by high concentrations of phage $\beta$. After purification, it produced target-like plaques on strain Davis, closely resembling those o phage $\beta$. Phage $\gamma^{\prime}$ is a clear-plaque mutant of phage $\beta^{\prime}$ in whose stocks it is always present.

The effect of the phages on various indicator strains is shown in Table 1. It will be seen that there was no cross-ir munity between phages $\beta$ and $\beta^{\prime}$.

\section{Cemmon characters}

The five phages shared many characters.

Morphology. Phages $\alpha, \gamma$ and $\gamma^{\prime}$ had the same morphology when examined by electron microscopy, which was kindly performed by Dr E. Kellenberger and Dr W. Arber (Pl. 1, fig. 1). Spe simens prepared by the agar-filtration method (Kellenberger \& Arber, 1957) and shadowed with a gold + platinum + palladium 
Table 1. Phage sensitivities of derivatives of Bacillus anthracis strain Davis

\begin{tabular}{|c|c|c|c|c|c|}
\hline \multirow[b]{2}{*}{ B. anthracis } & \multicolumn{5}{|c|}{ Phages } \\
\hline & $\beta$ & $\gamma$ & $\alpha$ & $\beta^{\prime}$ & $\gamma^{\prime}$ \\
\hline Davis & 1 & 1 & $\mathbf{1}$ & 1 & 1 \\
\hline Davis $(\beta)$ & C, $4 \times 10^{6}$ & $\mathrm{C}, 5 \times 10^{5}$ & eop 0.5 & $\operatorname{eop} 0 \cdot 7-1$ & eop $0 \cdot 7-1$ \\
\hline Davis $\left(\beta^{\prime}\right)$ & $\operatorname{eop} 1 \uparrow$ & eop 1 & eop 1 & . & C, $2.5 \times 10^{6}$ \\
\hline Davis $(\beta)\left(\beta^{\prime}\right)$ & . & . & eop 0.5 & . & C, $4 \times 10^{6}$ \\
\hline Davis $\left(\beta^{\prime}\right)(\beta)$ & . & . & eop 0.5 & . & $\mathrm{C}, 4 \times 10^{6}$ \\
\hline Davis/str (1) & eop 1 & $\operatorname{eop} 1$ & eop 1 & $\mathrm{C}, 4 \times 10^{8}$ & $\mathrm{C}, 4 \times 10^{6}$ \\
\hline
\end{tabular}

$\mathrm{C}=$ clearing only by high titre phage, followed by the smallest number of plaque-forming particles (pfp) in a $0.02 \mathrm{ml}$. drop found to give perceptible thinning of the bacterial lawn.

$\dagger$ eop $=$ efficiency of plating with reference to number of plaques produced on Bacillus anthracis strain Davis.

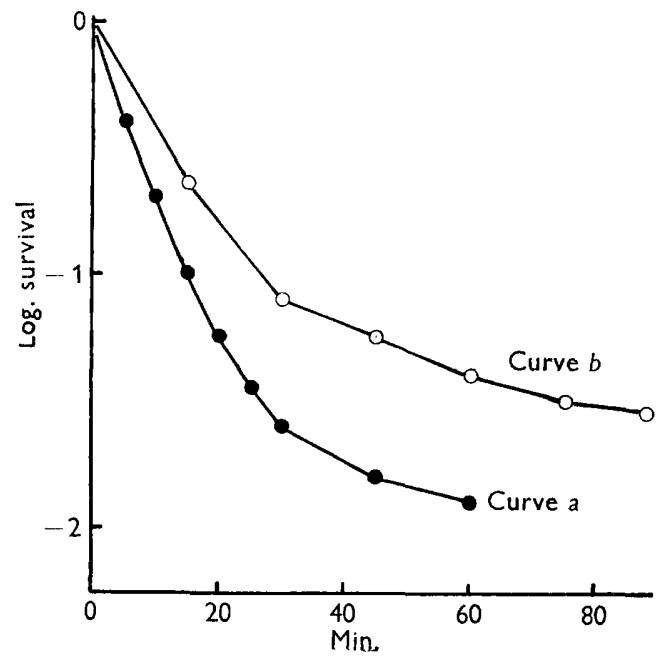

Fig. 1

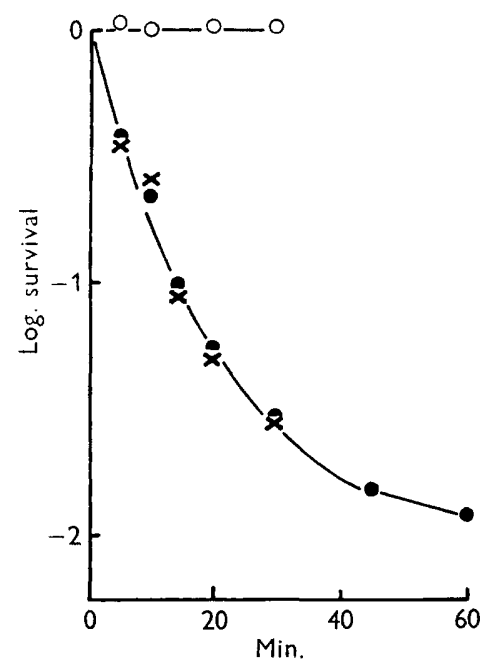

Fig. 2

Fig. 1. Rates of inactivation at $55^{\circ}$ in broth or in an inorganic salt solution. Curve $a$ : phages $\alpha, \beta$ and $\gamma^{\prime}$ in broth at initial concentrations of $1.4 \times 10^{6}, 2 \times 10^{6}$ and $1.2 \times 10^{6} \mathrm{pfp} / \mathrm{ml}$., respectively. Curve $b$ : phages $\alpha$ and $\gamma^{\prime}$ in an organic salt solution, composed of: $\mathrm{KH}_{2} \mathrm{PO}_{4}$, $10^{-2} \mathrm{M} ;\left(\mathrm{NH}_{4}\right)_{2} \mathrm{SO}_{4}, 1.5 \times 10^{-3} \mathrm{M} ; \mathrm{MgSO}_{4} .7 \mathrm{H}_{2} \mathrm{O}, 8 \times 10^{-5} \mathrm{M} ; \mathrm{Ca}\left(\mathrm{NO}_{3}\right)_{2}, 6 \times 10^{-6} \mathrm{M} ; \mathrm{FeSO}_{4}$, $2 \times 10^{-7} \mathrm{M} ; \mathrm{pH} 7$; at initial concentrations of $2 \times 10^{6}$ and $1.2 \times 10^{6} \mathrm{pfp} / \mathrm{ml}$, respectively. The sets of points for each curve overlapped too much for them to be shown separately.

Fig. 2. Phenotypic resistance to heat inactivation. Phage $\alpha$ at a concentration of $1.5 \times 10^{8} \mathrm{pfp} / \mathrm{ml}$. in broth was heated at $55^{\circ}$. After $60 \mathrm{~min}$. when only the resistant fraction remained, the suspension was removed and divided into two parts. Bacillus anthracis strain Davis was added to one part and after $15 \mathrm{~min}$. at $37^{\circ}$ to allow phage absorption, the suspension was centrifuged, the supernatant fluid containing unabsorbed phage was discarded, and the bacterial deposit resuspended in fresh broth. This was incubated at $37^{\circ}$ for $60 \mathrm{~min}$. and then centrifuged to separate the bacteria from the supernatant fluid containing newly liberated phage. The total time from addition of bacteria to separation of new phage was 100 min., which would allow no more than one cycle of phage multiplication. The rate of inactivation at $55^{\circ}$ of the new phage $(-x-)$ was then compared with that of the original suspension (--) and with that of the control portion of the original heat-resistant fraction (-O-). 
alloy showed phage particles with heads of $600 \AA$ diameter and tails $2000 \AA$ long and 100-150 $\AA$ broad. Many objects resembling detached tails were seen, and also flattened spheres, possibly collapsed heads.

Serology. Antisera for phages $\alpha, \beta, \gamma$ and $\gamma^{\prime}$ inactivated all four phages equally efficiently. None of the inactivation curves was exponential for each was initially concave (Fig. 6). This was not due to depletion of serum since fresh phage added to serum-phage mixtures in which the rate of inactivation had decreased was inactivated in the same way as the original phage.

Heat stability. Phages $\alpha, \beta, \gamma$ and $\gamma^{\prime}$ were inactivated similarly in broth at $55^{\circ}$ (Fig. 1, curve $a$ ), $56^{\circ}, 58^{\circ}$ and $59^{\circ}$, about $1.5 \%$ of phage appearing resistant at each temperature. The initial inactivation rate decreased and the size of the resistant fraction increased when phages $\alpha$ and $\gamma^{\prime}$ were heated in an inorganic salt mixture (Fig. 1, curve $b$ ). The resistant fraction consisted of phenotypically (Adams, 1953a) not genotypically, heat-resistant phage since its progeny had the same resistance as the initial population after only one cycle of multiplication (Fig. 2). Phenotypic resistance was not due to interaction between phage particles since the inactivation rate was the same at phage concentrations of $10^{8}$ and $10^{6}$ plaque-forming particles $(\mathrm{pfp}) / \mathrm{ml}$. Also, fresh phage added to a suspension, which had been heated until only the resistant fraction remained, was inactivated in the same way as the original phage.

Resistance to ultraviolet irradiation. All phages were inactivated at the same rate when plated on a non-lysogeric indicator (i.e. Bacillus anthracis strain Davis). However, the plaque counts were higher when u.v.-irradiated phage $\alpha$ was plated on indicators lysogenized by phage $\beta$ (McCloy, 1958) or phage $\beta^{\prime}$; this is another point of resemblance between these phages (Fig. 3).

One-step growth curve. Phages $\alpha, \beta, \gamma$ and $\gamma^{\prime}$ behaved similarly in experiments with Bacillus anthracis strain Davis in broth at $37^{\circ}$. The latent period was 65-70 min.; after this, the number of plaques increased for more than $3 \mathrm{hr}$. In some experiments, a plateau appeared at about 100-130 min. (Fig. 4) when the numbers of plaques suggested a burst size of 70-200. The shape of the curve suggested that it covered more than one cycle of multiplication although the chain concentration was very low $(50 \mathrm{ml}$.) and, since the experiments were made with chains initially containing only one infected bacterium, the neighbouring uninfected bacteria of the same chain were probably infected by the newly released phage of the first burst. No amount oî dilution could prevent this from happening. In experiments where no plateau was observed, the rise period of the first cycle was probably so prolonged that it merged with the rise period of the second.

Host range on naturally occurring strains. Phage $\alpha:(a)$ attacks all strains of Bacillus anthracis (McCloy, 1951). It has since been used routinely in the Public Health Laboratory, County Hall, London, for over 9 years in the routine identification of anthrax strains, over 800 having been isolated in this period (Dr A. J. H. Tomlinson, personal communication); (b) attacks about $4 \%$ of strains of $B$. cereus; and $(c)$ does not attack strains of other Bacillus species.

Phages $\beta$ and $\gamma$ had the same host range as phage $\alpha$ in limited tests (neither forms plaques on strains lysogenized by phage $\beta$ ).

Phage $\gamma^{\prime}:(a)$ does not attack any strain of Bacillus anthracis or $\boldsymbol{B}$. cereus resistant to phages $\alpha, \beta$ and $\gamma$ and only multiplied in 4/95 strains sensitive to 
those phages (Table 2). On most of the remainder its lysates produced colicine-like clearing; i.e. partial clearing with high concentrations which disappeared rapidly on dilution; $(b)$ has not been tested on other Bacillus species.

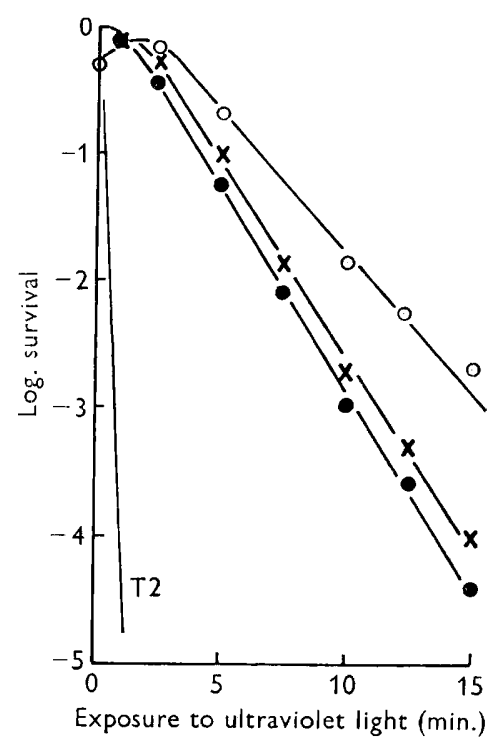

Fig. 3

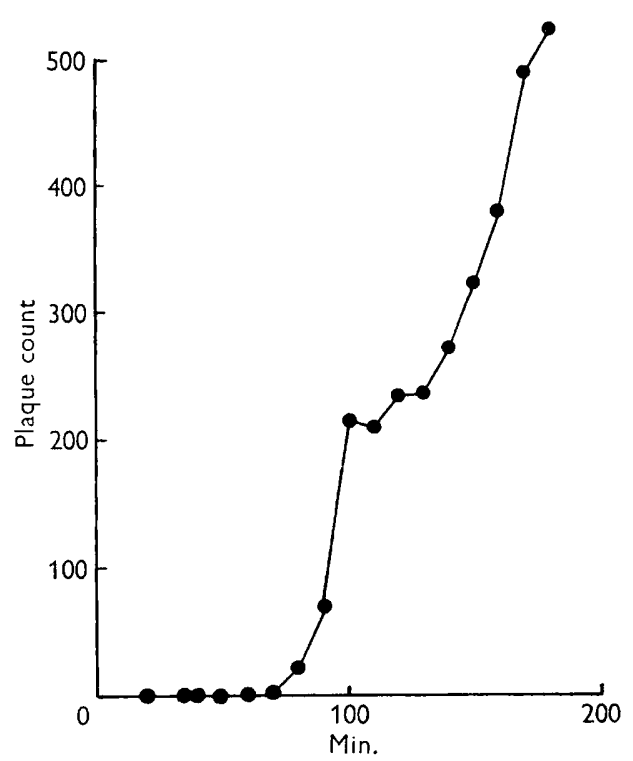

Fig. 4

Fig. 3. Inactivation of phage $\alpha$ and phage $\gamma^{\prime}$ by ultraviolet radiation. Reactivation of u.v.-inactivated phage $\alpha$ in Davis $\left(\beta^{\prime}\right)$. Samples of phage $\alpha(--)$ and phage $\gamma^{\prime}(-0)$ were plated with $B$. anthracis strain Davis; and samples of phage $\alpha$ were also plated with B. anthracis strain Davis $(\beta \mathrm{LC})\left(-\mathrm{O}^{-}\right)$and $B$. anthracis Davis $\left(\beta^{\prime}\right)(-\times-)$. The points for the first two curves overlapped too much for them to be drawn separately. (-) Inactivation curve of coliphage $\mathrm{T} 2$.

Fig. 4. One-step growth experiment with phage $\alpha$ growing on Bacillus anthracis strain Davis. Phage $\alpha$ at $4.7 \times 10^{6} / \mathrm{ml}$. was mixed in broth at $37^{\circ}$ with strain Davis (turbidity $\approx$ Escherichic coli at $5 \times 10^{8} / \mathrm{ml}$.). After allowing $5 \mathrm{~min}$. for adsorption, the mixture was diluted so that the concentration of pfp was $458 / \mathrm{ml}$. in the tubes from which the initial counts were made. The pfp counts, expressed as multiples of the initial number, are plotted against the time elapsed since phage and bacteria were mixed.

Host range on artificial phage-resistant strains. All the phages attacked Bacillus anthracis strain Davis but both partially and then completely resistant variants could be isolated (see Methods). Those of either sort selected by either phage $\alpha, \gamma$ or $\gamma^{\prime}$ were equally resistant to all five phages: no detectable adsorption occurred.

Another type of phage-resistant strain was also encountered. This was Bacillus anthracis strain Davis/str (1), a streptomycin-resistant mutant of strain Davis which had never been in contact with phage and resembled its parent in its cultural characteristics, but on which phage $\gamma^{\prime}$ failed to grow although it produced colicinelike clearing (Table 1). The efficiency of plating (e.o.p.) of either phage $\alpha, \beta$ or $\gamma$ was 1 on this strain. All the other streptomycin-resistant mutants isolated were sensitive to phage $\gamma^{\prime}$.

Adsorption. None of the phages adsorbed to phenotypically capsulated organisms of any strain. Nor did they adsorb well to organisms that were non-capsulated 
(whether following mutation cr cultivation in an environment unsuitable for capsule formation (Sterne, 1937)) if the strains were morphologically and culturally typical. But all phages adsorbed rapidly to smooth or RS colony variants (Nungester, 1929). For example, 9/14 typical strains absorbed less than $10 \%$, and the remaining 5 strains $30-60 \%$, of the phage in $30 \mathrm{~min}$; but atypical S or RS variants isolated from 11 of these bacterial strains absorbed more than $90 \%$ within 10 min.

Table 2. Sensitivities of strains of Bacillus anthracis and Bacillus cereus to high titre preparations of phages $\gamma$ and $\gamma^{\prime}$

Each bacterial strain was spread over half a nutrient agar plate and $0.02 \mathrm{ml}$. drops of phage $\gamma$ and phage $\gamma^{\prime}$ containing $10^{8}$ pfp were spotted on them. The degree of clearing was recorded as : $\infty$ complete; +++ only a thin veil of growth $;++$ marked; + just perceptible (often only visible when viewed obliquely); - no perceptible clearing. On two strains of $B$. cereus the high-titre phage produced only a few isolated plaques.

The degree to which a strain was cleared by phage $\gamma^{\prime}$ depended markedly on the size of the bacterial inoculum; the results given here were those obtained with inocula just large enough to give a confluent bacterial lawn.

Phage dilutions containing $2 \times 10^{3} \mathrm{pfp} / \mathrm{drop}$ were also tested: with phage $\gamma$, all the strains markedly cleared by concentrated phage $(++$ or more) showed semi-confluent plaques; with phage $\gamma^{\prime}$ only three strains of $B$. anthracis and one of $B$. cereus showed any clearing.

\section{Bacillus anthracis}

\section{Degree of clearing} by phage $\gamma$

$$
\begin{aligned}
& \infty \\
& ++t \\
& ++
\end{aligned}
$$$$
+
$$$$
-
$$

Total

$\infty$
4
0
0
0
0
4

Degree of clearing by phage $\gamma^{\prime}$

$\begin{array}{rrrrr}++ & ++ & + & - & \text { Total } \\ 4 & 19 & 19 & 4 & 50 \\ 0 & 11 & 14 & 1 & 26 \\ 0 & 2 & 8 & 0 & 10 \\ 0 & 0 & 7 & 0 & 7 \\ 0 & 0 & 0 & 0 & 0 \\ 4 & 32 & 48 & 5 & 93\end{array}$

Bacillus cereus

Degree of clearing by phage $\gamma^{\prime}$

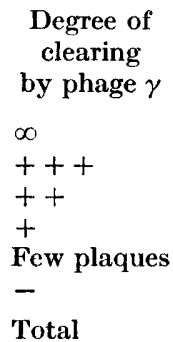

$\begin{array}{ccccccc}\infty & +++ & ++ & + & \begin{array}{c}\text { Few } \\ \text { plaques }\end{array} & - & \text { Total } \\ 0 & 0 & 0 & 0 & 0 & 0 & 0 \\ 0 & 1 & 0 & 0 & 0 & 0 & 1 \\ 0 & 0 & 0 & 1 & 0 & 0 & 1 \\ 0 & 0 & 0 & 0 & 0 & 0 & 0 \\ 0 & 0 & 0 & 0 & 2 & 0 & 2 \\ 0 & 0 & 0 & 0 & 0 & 47 & 47 \\ 0 & 1 & 0 & 1 & 2 & \mathbf{4 7} & \mathbf{5 1}\end{array}$

Differences in adsorption probably account for the finding that phages $\alpha$ and $\gamma$ cleared broth cultures of smooth but not of typical strains, and produced clear 'punched-out' plaques on smooth strains but only shallow depressions on typical ones (which might have a tiny central clear spot). Also, these differences may explain why the concentration of phage $\gamma^{\prime}$ needed to produce a colicine-like effect was 10-100 times greater with a typical than with a smoother strain. These observations suggest that variation to the smoother colony form causes the phage receptor to become a more prominent component of the bacterial surface. 
Phages $\gamma$ and $\gamma^{\prime}$ adsorbed equally well to the 11 atypical variants described above and to strain Davis and all its variants including Davis/str (1) (except for the completely resistant mutants selected by phages $\alpha, \gamma$ or $\gamma^{\prime}$ discussed in the previous section) even when phage $\gamma^{\prime}$ did not subsequently multiply. Thus there was nothing to indicate that phages $\gamma$ and $\gamma^{\prime}$ attached to different receptors on the bacterial surface.

Induction of lysogenic strains following superinfection. When either phage $\alpha$ or phage $\gamma$ infected strain Davis $(\beta)$, vegetative multiplication of the prophage was induced (McCloy, 1958). Similarly, phage $\gamma^{\prime}$ induced strain Davis $\left(\beta^{\prime}\right)$. Phage $\alpha$ and phage $\gamma$ also induced prophage $\beta^{\prime}$ though they had less effect on this prophage than on prophage $\beta$.

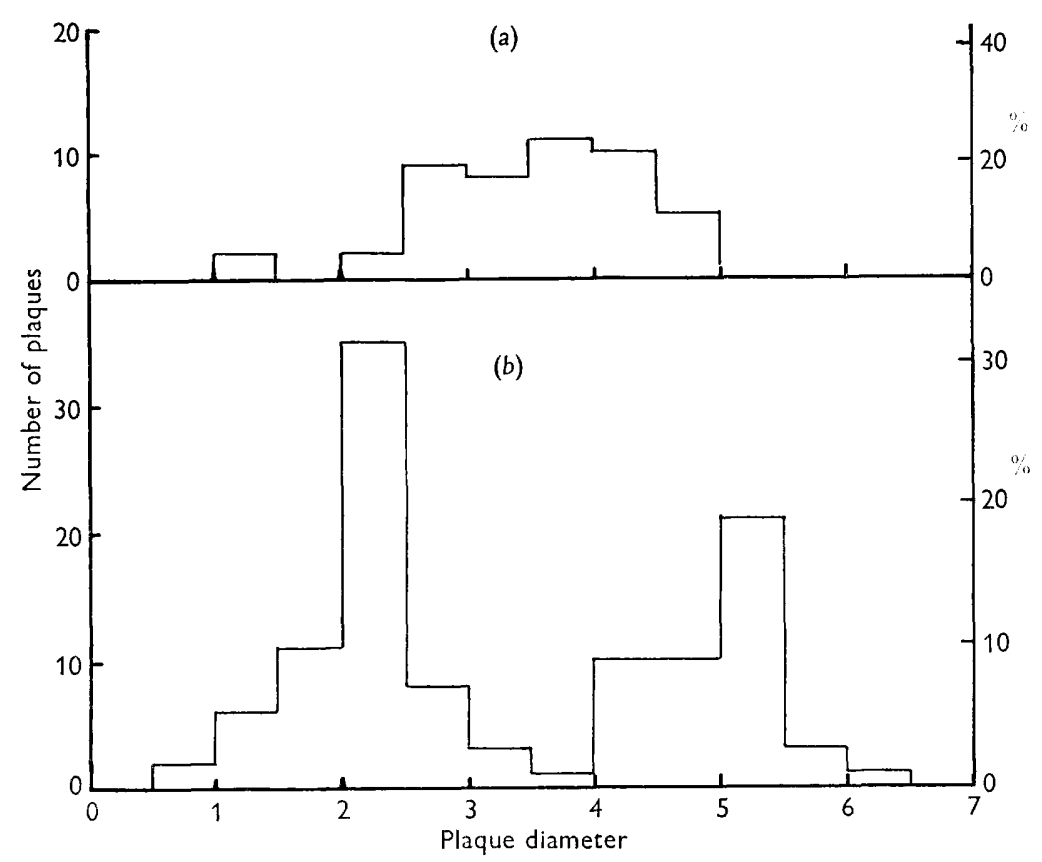

Fig. 5. The distribution of plaque diameters for normal and serum-treated stocks of phage $\alpha$. 'The numbers on the abscissa are the diameters $(\mathrm{mm}$.) as measured on photographs of plates enlarged $2 \cdot 4$ times. The numbers on the left-hand ordinate are the numbers of plaques counted/class, the corresponding \% being shown on the right-hand ordinate. Fig. 5a. Normal phage $\alpha$, showing a unimodal distribution, measured from the plate shown in Pl. 1, fig. 2. Fig. $5 b$. Phage $\alpha$, plated after exposure to phage $\beta$ anti-serum when the total survival was $8 \%$, showing a bimodal distribution. The measurements were taken from the plate shown in Pl. 1, fig. 3. The mean plaque diameter differed from plate to plate: hence, the mean of Fig. 5 a does not correspond to the class of large plaques in Fig. $5 b$.

\section{Plaque size during serum inactivation}

The distribution of plaque diameters in ordinary phage stocks was unimodal (Fig. $5 a$; PI. 1, fig. 2) but during serum inactivation became bimodal owing to the appearance of a class of unusually small plaques (Fig. $5 b$; Pl. 1, fig. 3). The small plaques were directly due to the action of the antiserum, for their absolute concen- 
tration increased with exposure of the phage to serum (Fig. 6). The small plaques yielded phage which gave normal-sized plaques on replating and which was neutralized in the same way as the original phage. Presumably, therefore, they arose from particles whose growth on the indicator was delayed. This was not likely to be due to reactivation of completely neutralized particles following either spontaneous dissociation of phage and antibody (Jerne \& Avegno, 1956) or digestion of antibody by proteolytic enzymes produced by the indicator strain (see Weiss, 1927; Kalmanson \& Bronfenbrenner, 1943), for the titre of an antiserum was not decreased by incubation with cultures of strain Davis, and storage of serum-inactivated phage in either broth or culture filtrates of strain Davis for as long as $9 \mathrm{hr}$. did not alter the number of plaque-forming particles or the ratio of small/large plaques. The small

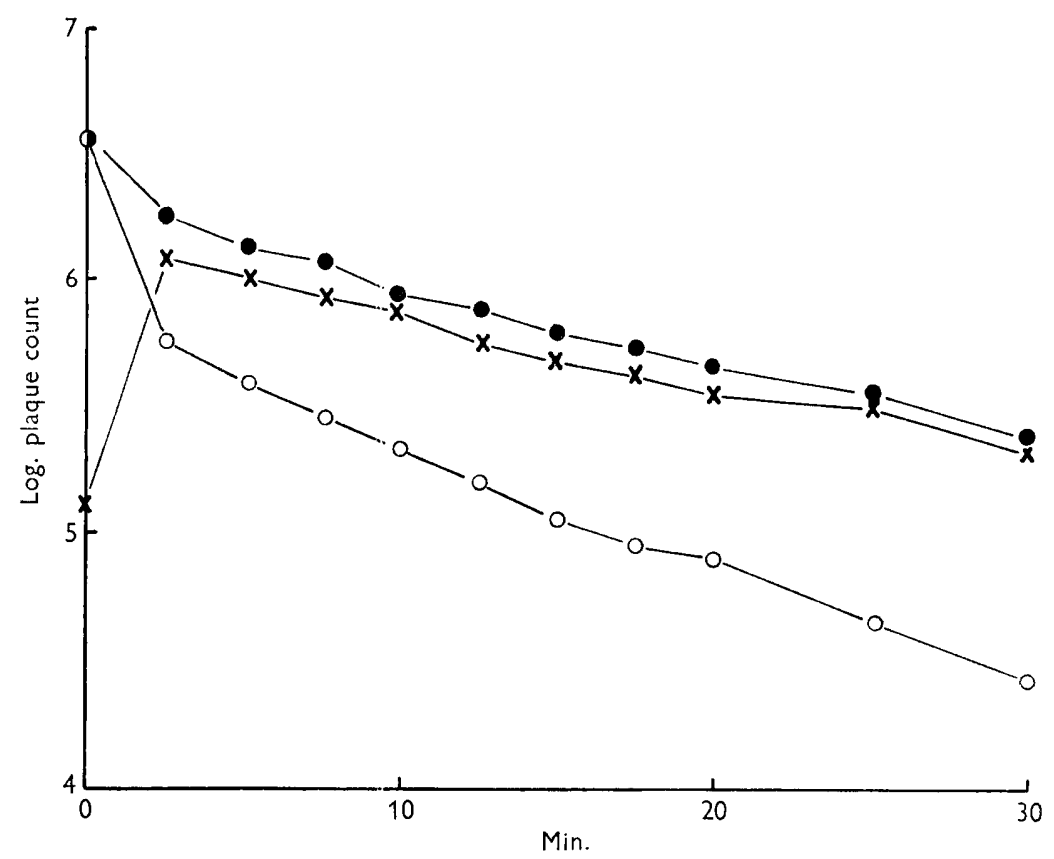

Fig. 6. Serum inactivation curves, showing the changes in the concentrations of small $(-\times-)$ and normal (-O-) plaques as well as the total number of plaques (- - ).

plaques were therefore thought to be formed by 'incompletely neutralized' particles (Burnet, Keogh \& Lush, 1937; Andrewes \& Elford, 1933) „which might adsorb more slowly than normal to the indicator bacteria, or be delayed at some later stage such as injection of the phage DNA (Nagano \& Oda, 1955). In fact, both processes were involved (Table 3), for about half the particles began to multiply late even after adsorption had occurred. Control experiments showed that when plates were spread with indicator bacteria and incubated for various periods before phage was inoculated, the resultant plaques only became small when phage was added $5 \mathbf{- 6} \mathrm{hr}$. after the bacteria. The doubling time of strain Davis is about $1 \mathrm{hr}$.: thus it seemed that the serum-treated particles which produced small plaques could be attached to bacteria for at least 5-6 generations before injection occurred.

Complete inactivation could probably occur either in one step or after partial 
inactivation, since examination of the initial part of the inactivation curve showed that the total plaque count decreased before the count of small plaques increased. The relative importance of the two types of inactivation can be estimated by determining the inactivation curves for normal, small and total plaques in ideal conditions in which the phage particles are equally susceptible to inactivation and the inactivation curves are ultimately exponential. The concavity of the present curves (Fig. 6) showed this sort of analysis to be impossible in the present system. However, since these experiments were done, a purely two-step inactivation process has been shown in this way by Graziosi, Coppo \& Donini (1954) with a phage of Bacillus megaterium.

Table 3. To show the decreased rate of adsorption and delay in infection by particles forming small plaques after exposure to anti-phage serum

Phage $\gamma$ at a concentration of $4.5 \times 10^{7}$ p.f.p./ml. was mixed with an anti- $\gamma$ serum diluted 1/200. After $20 \mathrm{~min}$. at $37^{\circ}, 1 \mathrm{ml}$. was added to $9 \mathrm{ml}$. broth (sample $a$ ) from which $2 \mathrm{ml}$. was at once mixed with $2 \mathrm{ml}$. broth suspension of strain Davis (turbidity $\approx 2 \times 10^{9}$ Escherichia coli/ml.). After $10 \mathrm{~min}$. at $37^{\circ}$ (sample $b$ ), $1 \mathrm{ml}$. was added to $9 \mathrm{ml}$. broth and centrifuged. The supernatant fluid was removed (sample $c$ ) and the deposit washed once with $10 \mathrm{ml}$. broth (sample $d$ ).

Samples $a-d$ were plated with Bacillus anthracis strain Davis and the numbers of normal and small plaques recorded with the following results.

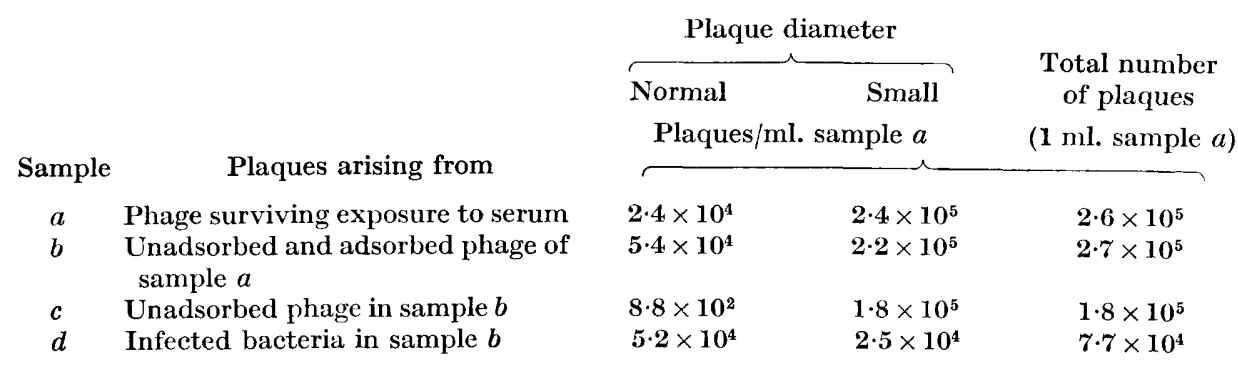

The Table shows that:

(1) $96 \%$ of the particles forming normal plaques were adsorbed (comparison of samples $a$ and $c$ ). Particles forming small plaques adsorbed too slowly for their number to be calculated directly in the same way but it could be obtained as follows :

(2) the numbers of normal plaques was higher in sample $b$ than in $a$. This difference of $3 \times 10^{4}$ must have been due to adsorption of particles forming small plaques in the free state which formed normal plaques when plated after adsorption. $3 \times 10^{4}$ is $12 \%$ of the initial number of small plaque-forming particles in sample $a$;

(3) the infected bacteria (sample $d$ ) produced $2.5 \times 10^{4}$ small plaques. These must have been produced by bacteria infected by particles forming small plaques (indicating a delay in infection after adsorption);

(4) the total number of small plaque-forming particles adsorbed was therefore $3 \times 10^{4}+2.5 \times 10^{4}$ $=5 \cdot 5 \times 10^{4}=23 \%$ of the initial number in sample $a$. This is markedly less than the value of $96 \%$ observed for particles forming normal plaques.

\section{Plating of phage $\alpha$ on different indicators}

Host-induced phenotypic modification (Bertani \& Weigle, 1953) of phage $\alpha$. Phage $\alpha$ could exist in two forms: one, induced by growth in the naturally lysogenic Bacillus cereus strain W, had a high e.o.p. on this strain ; and the other induced by growth in any other strain had a low e.o.p. on strain W. The difference did not lie between $B$. cereus and $B$. anthracis nor between lysogenicity and non-lysogenicity with phage $\beta$ : the phage was modified in the same way whether it had grown on non-lysogenic 
strains of $B$. anthracis, a strain of $B$. cereus, or on derivatives of these strains lysogenic with $\beta$. None of these strains had the specific resistance of strain $W$ to the phage grown on other strains. With preparations grown on strains other than $W$, the ratio of plaques produced on strain $W$ to plaques on strain Davis was $10^{-5}$ to $10^{-6}$ (Table 4, col. c). Phage isolated from the rare plaques on strain $\mathrm{W}$ was indistinguishable from other preparations of $\alpha$ grown on $W$ in having a high e.o.p. on strain $W$ which again decreased when it was grown on other strains; a single cycle in strain Davis was enough to produce the change. Phage $\alpha$ grown on $W$ had a relatively high e.o.p. on strain $\mathbf{W}$, but, unlike preparations grown on other strains, produced substantially different numbers of plaques on different strains, the greatest number being on strain $2160 \mathrm{~S}$ (Table 4, col. a).

Table 4. Relative efficiencies of plating of phage $\alpha$ on different Bacillus strains

\begin{tabular}{|c|c|c|c|c|}
\hline $\begin{array}{l}\text { Bacillus } \\
\text { strain }\end{array}$ & $\begin{array}{c}(a) \\
\alpha / W\end{array}$ & $\begin{array}{c}(b) \\
\alpha 2 ; W\end{array}$ & $\begin{array}{c}(c) \\
\alpha / \text { other than W }\end{array}$ & $\begin{array}{c}(d) \\
\alpha 2 / \text { other than W }\end{array}$ \\
\hline $2160 \mathrm{~S}$ & 1 & 1 & $\mathbf{1}$ & 1 \\
\hline $2160(\beta) \mathrm{S}$ & $0 \cdot 6-0 \cdot 8$ & $0.6-.0 \cdot 8$ & $0.5-0.75$ & $0.5-0.75$ \\
\hline$W$ & $0 \cdot 2$ & $0 \cdot 8$ & $1 \times 10^{-6}-1 \times 10^{-5}$ & $4 \times 10^{-5}-1 \times 10^{-4}$ \\
\hline Davis & $0 \cdot 03^{*}$ or $0 \cdot 3$ & 0.08 & 1 & 1 \\
\hline Davis $(\beta)$ & $0 \cdot 015$ & 0.015 & $0 \cdot 5-0.75$ & $0.5-0.75$ \\
\hline 1444 & $0 \cdot 01$ & . & $0 \cdot 75-1$ (indistinct) & . \\
\hline 2160 & $0 \cdot 003$ & 0.003 & c. 0.8 (indistinct) & - \\
\hline
\end{tabular}

* The efliciency of plating on B. anth acis strain Davis of phage $\alpha$ grown on B. anthracis strain W was always $0.02-0.05$ when the plating bacteria were taken from actively growing cultures; but when they were taken from plates which had been incubated for $24 \mathrm{hr}$. it sometimes increased to about $0 \cdot 3$.

Phage $\beta$ was host-modified in the same way as phage $\alpha$ for, while preparations of $\beta$ grown on Davis produced the same numbers of plaques on strain $2160 \mathrm{~S}$ as on strain Davis, phage $\beta$ in supernatants of cultures of strain $W$ produced only $1 / 50$ as many plaques on strain Davis as on strain $2160 \mathrm{~S}$.

Phage $\gamma$ was evidently also host-modified for when it was grown on strain Davis it plated with equal efficiency on strains Davis and $2160 \mathrm{~S}$, but supernatant fluids from cultures of strain $W$ produced fewer $\gamma$ plaques on strain Davis than on strain $2160 \mathrm{~S}$.

Plaques on lysogenic indicators: (a) lysogenic derivatives of the indicators Bacillus anthracis strains Davis and $2160 \mathrm{~S}$. Two plaque-type variants of phage $\alpha$ were noted in the following way. 'The plaques formed by phage $\alpha$ on the non-lysogenic strains, Davis and $2160 \mathrm{~S}$, are relatively large (of diameter $3 \mathrm{~mm}$. on strain Davis) whereas those formed by ordinary stocks of phage $\alpha$ on the same indicators lysogenized by either $\beta$ or $\beta$ LC are all small (of diameter $1 \mathrm{~mm}$. on lysogenic Davis (Pl. 2, fig. 4)) . When these small plaques were replated with lysogenic Davis or lysogenic $2160 \mathrm{~S}$, large as well as small plaques were formed (PI. 2, fig. 5). Phage $\alpha$ isolated from the large plaques continued to produce large plaques after propagation on non-lysogenic (or lysogenic) strains.

The phage which produced large plaques on Davis $(\beta)$ and $2160 \mathrm{~S}(\beta)$, designated $\alpha \mathrm{L}$, was presumably a mutant of the phage which produced small plaques, $\alpha \mathrm{S}$, selected by growth on the lysogenic strains. It was unlikely to have arisen following recombination between $\alpha \mathrm{S}$ and the prophage in respect of a plaque-type character, 
for $\beta$ and $\alpha$ are probably identical save for a single mutation which determines the virulence of phage $\alpha$. The proportions of $\alpha \mathrm{L}$ in plaques produced by particles of $\alpha \mathrm{S}$ depended on the strain; high proportions were observed with lysogenic Davis, and less with lysogenic $2160 \mathrm{~S}$ and $\mathrm{W}$ (Table 5). No $\alpha \mathrm{L}$ was ever observed when $\alpha \mathrm{S}$ was grown in non-lysogenic strains. The higher proportion of $\alpha \mathbf{L}$ with Davis $(\beta)$ than with $2160 \mathrm{~S}(\beta)$ or $\mathrm{W}$ was due to the strain of bacterium and not to the prophage $(\beta)$ it carried. Table 5 shows that the proportion of $\alpha \mathbf{L}$ was still high with strain Davis lysogenized with phage $\beta$ derived from either strain $2160 \mathrm{~S}(\beta)$ or the naturally occurr-

Table 5. Percentage of $\alpha L$ in plaques produced by $\alpha S$ on lysogenic strains

Phage $\alpha S / D a v i s$ was plated with the lysogenic strain, and individual plaques were tested for the proportions of phages $\alpha \mathrm{S}$ and $\alpha \mathrm{L}$ which they contained by plating samples with streptomycin-resistant $B$. anthracis Davis $(\beta \mathrm{LC})$ on streptomycin agar. The samples were diluted to allow the observation of 100-500 plaques.

$\begin{array}{lccccccc}\text { Bacillus strain } & \begin{array}{c}\text { None } \\ \text { seen }\end{array} & \begin{array}{c}\text { 1 or } \\ \text { less }\end{array} & 2-20 & 21-40 & 41-60 & 61-80 & 81-100 \\ \text { Davis }(\beta) & . & 1 & 8 & 6 & 6 & 3 & 8 \\ \text { Davis }(\beta)\left(\beta^{\prime}\right) & 2 & 8 & 18 & 5 & 8 & . & 11 \\ \text { Davis }(\beta L C) & 1 & 2 & 10 & 1 & 10 & 4 & 12 \\ 2160 S(\beta) & 8 & 12 & 2 & . & . & . & \text {. } \\ \text { W } & 6 & 3 & 1 & . & . & . & \text {. }\end{array}$

Isolate
1
2
3
4
5
6
7
8
9
10
11
12

Davis lysogenized with $\beta$ from $2160 \mathrm{~S}(\beta)$

Isolate 1
$\mathbf{2}$
$\mathbf{3}$
$\mathbf{4}$
$\mathbf{5}$

Davis lysogenized with $\beta$ from $W$

Isolate 1
2
3
4

2160S lysogenized with $\beta \mathrm{LC}$ from Davis $(\beta \mathrm{LC})$

Davis $\left(\beta^{\prime}\right)$

$\begin{array}{lll}1 & 3 & 1 \\ 2 & 2 & 1 \\ 3 & 2 & \text {. } \\ 2 & 1 & 1\end{array}$

30

ing lysogenic strain, $\mathrm{W}$; and was still low with strain $2160 \mathrm{~S}$ lysogenized with either phage $\beta$ or phage $\beta \mathrm{LC}$ derived from strain Davis $(\beta)$ or strain Davis $(\beta \mathbf{L C})$ respectively. Phage $\alpha \mathrm{L}$ also appeared when phage $\alpha \mathrm{S}$ was grown on strain Davis $(\beta)$ in broth, but did not form as high a proportion of the total phage as in plaques on solid medium. 
The only difference observed between phages $\alpha \mathrm{L}$ and $\alpha \mathrm{S}$ growing in Davis ( $\beta \mathbf{L C}$ ) that might account for a selective advantage of $\alpha \mathbf{L}$ was that plaques were produced by the infected bacteria on streptomycin agar earlier with phage $\alpha \mathbf{L}$, suggesting that mature phage might be formed earlier with $\alpha \mathbf{L}$ than with $\alpha \mathrm{S}$. This difference was more marked with stationary phase $(\mathbf{2 4} \mathrm{hr}$. broth cultures) than with actively growing bacteria. The phages adsorbed equally well to Davis $(\beta \mathbf{L C})$, and in one-step growth experiments with actively growing bacteria, they had the same minimum latent period, after which they both increased at the same rate. Both phages led to the production of the same amount of the temperate phage.

Strain Davis lysogenized by phage $\beta^{\prime}$ behaved in these experiments like a nonlysogenic strain, i.e. phage $\alpha \mathrm{S}$ produced large plaques on Davis $\left(\beta^{\prime}\right)$ which did not contain any $\alpha \mathrm{L}$ in tests on Davis ( $\beta \mathrm{LC}$ ) (Table 5 ). The doubly lysogenic $B$. anthracis strain Davis $\left(\beta^{\prime}\right)(\beta)$ behaved like Davis $(\beta)$. All preparations of phage $\gamma^{\prime}$ examined produced large plaques on Davis $(\beta \mathrm{LC})$.

Bacillus cereus strain $W$. Ordinary stocks of phage $\alpha \mathrm{S}$ or phage $\alpha \mathrm{L}$ formed turbid plaques on Bacillus cereus strain $W$ containing $\alpha$-sensitive bacteria ( $\mathrm{Pl}$. 2, fig. 3). Occasional clear areas could be seen which contained phage, designated $\alpha 2$, which produced much clearer plaques on B. cereus strain W (Pl. 2, fig. 7). Secondary colonies appearing after lysis by phage $\alpha \mathbf{2}$ were all resistant to phage $\alpha$ (and to $\alpha \mathbf{2}$ ). The plaques of ordinary phage $\alpha$ and phage $\alpha 2$ were equally heterogeneous in size, and both phages adsorbed extrenely slowly to B. cereus strain W. However, a difference was observed when infected bacteria were plated instead of free phage, for phage $\alpha 2$ then produced large plaques whereas those of ordinary phage $\alpha$ were as heterogeneous as before. This may mean that infected bacteria burst earlier and more synchronously with phage $\alpha 2$ than with phage $\alpha$. Phage $\alpha 2$ was host-modified in the same way as phage $\alpha$ itself, but preparations in either form had an e.o.p. on strain W 5-10-fold higher than corresponding preparations of phage $\alpha$ (Table 4).

\section{Bacterial sporulation despite phage infection}

Bacteria infected with phages $\alpha$ or $\gamma$ formed spores which contained the phage in a heat-stable form and liberated it on plating. In lysogenic Bacillus strains, the prophage in spores is heat-resistant (Den Dooren de Jong, 1931 ; Cowles, 1931); and Adant(1932)

and Wollman '\& Wollman (1936) observed that what was evidently vegetatively multiplying phage present in the spores of Bacillus subtilis also survived heating. The fate of individual spores of $B$. cereus strain $\mathrm{W}$ formed in the presence of phage $\alpha$ could be determined because uninfected organisms of this strain produced characteristic colonies on lawns of the indicator $B$. anthracis strain Davis which could be easily distinguished and counted. When heated suspensions of well-separated spores taken from cultures of $\boldsymbol{B}$. cereus strain W infected with phage $\alpha$ were plated, individual spores evidently gave rise either to a colony or to a plaque, but not to both: sufficiently dilute suspensions produced plaques which were free from colonies of $\boldsymbol{B}$. cereus strain W, and colonies of $B$. cereus strain W free from phage $\alpha$.

The conclusion that individual spores of Bacillus cereus strain $\mathrm{W}$ gave rise either to a colony or to a plaque was supported by the results of a terminal dilution experiment in which the products of single spores were examined. This was made with a 
spore suspension which had produced $1.8 \times 10^{6}$ colonies and $5 \times 10^{5}$ plaques. When $1 \mathrm{ml}$. samples of a $10^{-7}$ dilution in broth were distributed and incubated for $4 \frac{1}{2} \mathrm{hr}$. before plating with the indicator culture, 10/60 samples gave only colonies and 4/60 gave only plaques; none gave colonies and plaques.

The experiments were repeated with a non-lysogenic strain, Bacillus anthracis strain 1444, infected with phage $\alpha$ and phage $\gamma$, and the same conclusion was reached as with $B$. cereus strain $W$, i.e. that the bacteria were still able to sporulate after infection by either phage, and that individual spores produced a colony or a plaque, but not both. The results with phage $\alpha$ were confused to some extent in that a proportion (about $0.5 \%$ ) of spores produced in the presence of this phage turned out to be lysogenic with phage $\beta$; none of the $\alpha$-lysogenic clones obtained by Ivanovics (1962) were observed in these experiments. Spores lysogenic with phage $\beta$ were found even when the suspensions were harvested from single plaques produced by phage $\alpha$ on $B$. anthracis strain 1444. The reason for this is not clear since phage $\beta$ was not detected either as free phage or as prophage in vegetative cells of strain Davis infected by phage $\alpha$. It is therefore possible that phage $\beta$, arising from phage $\alpha$ by mutation, was much more strongly selected for when sporulation occurred than during vegetative growth. No spores lysogenic with phage $\beta$ were observed when $B$. anthracis strain 1444 was infected with phage $\gamma$.

Fully formed heat-resistant spores of Bacillus anthracis strain 1444 could not take up phage $\gamma$ and make it heat-resistant, and no adsorption of this phage to spores was detected; thus phage $\gamma$ presumably infected vegetative bacteria and did not prevent subsequent sporulation. Perhaps infection did not interfere with the syntheses associated with sporulation (Hardwick \& Foster, 1952), since the conditions which favour sporulation differed so fundamentally from those which favour vegetative bacterial growth, and also presumably phage growth. Or, the spores which contained phage might have been derived from bacilli which, although they had the morphology and so presumably the phage receptors of vegetative bacilli, were in fact irreversibly committed to sporing (Foster \& Perry, 1954) by the time infection took place. No experiments were made to distinguish between these possibilities.

\section{DISCUSSION}

The characters of phages $\alpha, \beta$ and $\gamma$ were described in part in previous papers (McCloy, 1951, 1953, 1958). Phage $\beta^{\prime}$ was isolated only once so that it might have been a contaminant and not a mutant of phage $\beta$. However, not only did it resemble phage $\beta$, but its derivative, $\gamma^{\prime}$, also resembled phages $\alpha, \beta$ and $\gamma$ in many ways, e.g. in the size of the phenotypically heat-resistant fraction (Fig. 1), the appearance of small plaques during serum inactivation (Fig. 6), and the similar rates of inactivation (Figs. I, 6). Also other Bacillus phages isolated did not resemble phage $\beta$, so that the mutational origin of phage $\beta^{\prime}$ seems likely.

Despite the similarities of the five phages, including a common bacterial receptor, phage $\gamma^{\prime}$ had a much smaller host range than phages $\alpha, \beta$ and $\gamma$ ('Table 1). One of its hosts, Bacillus anthracis strain Davis, could mutate to a form (Davis/str (1)) resembling most naturally-occurring anthrax strains on which phage $\gamma^{\prime}$ had a colicinelike action but could not form plaques. Considering that these phages are so alike and that related phages can multiply simultaneously in the same bacterium (Adams, 
$1953 b$ ), it was expected that phages $\gamma$ and $\gamma^{\prime}$ would grow together in mixed infection, and, if so, that phage $\gamma$ might enable $\gamma^{\prime}$ to grow in hosts in which it would not grow alone. This was found to be so in all three strains tested (Davis/str (1), 2160 S, RVHS). Phage $\gamma^{\prime}$ could also grow in these strains after mutation.

The formation of abnormally small plaques during serum inactivation has been described before but, in general, the plaque diameter appears to decrease progressively as inactivation proceeds (Burnet et al. 1937; Andrewes \& Elford, 1933); whereas in this system, and in that studied by Graziosi et al. (1954), the average diameter of the small plaques remained constant throughout, despite differences in their number according to the extent of inactivation. The assay method probably detected all partially inactivated particles since the smallest of the small plaques was considerably larger than the minimum size of plaque visible in the lawn. It follows that the partially inactivated particles probably form a distinct class, subject to a certain delay in establishing infection.

It is well known that the prophage in spores of lysogenic strains can survive heating which inactivates the free phage (Den Dooren de Jong, 1931; Cowles, 1931). The present experiments as well as those of Adant (1932) and of Wollman \& Wollman (1936) suggest that vegetatively growing phage can also survive in heated spores, i.e. sporulation can occur despite vegetative phage multiplication. On germination, each spore of Bacillus cereus strain $W$ produces either a plaque or a colony free from phage (McCloy, 1953). This conclusion was almost certainly correct for B. cereus strain $W$ infected by phage $\alpha$, with which a terminal dilution experiment was done. It was probably also correct for the typical anthrax strain, 1444, infected by phages $\alpha$ or $\gamma$ although Ivanovics (1962) has since shown that such strains can be lysogenized by phage $\alpha$ and that lysogenic spores form either a plaque or a colony, depending on whether or not they are plated with indicator bacteria. This difficulty was avoided in the present experiment with $B$. cereus strain $W$ because colonies and plaques could be counted on the same plate.

\section{REFERENCES}

Adnus, M. H. (1953a). The genotypically and phenotypically heat resistant forms in the T5 species of bacteriophage. Ann. Inst. Pasteur, 84, 164.

Adams, M. H. (1953b). Criteria for a biological classification of bacterial viruses. Ann. N.Y. Acad. Sci. 56, 442.

Adant, M. (1932). Le bactériophage du Bacillus subtilis sporulé. C.R. Soc. Biol., Paris, $111,1055$.

Andrewes, C. H. \& Elford, W. J. (1933). Observations on anti-phage sera: II. Properties of incompletely neutralized phage. Brit. J. exp. Path. 14, 376.

Bertani, G. \& Weigle, J. J. (1953). Host controlled variation in bacterial viruses. J. Bact. 65, 113.

Bordet, J. \& Renaux, E. (1930). L'influence du calcium sur l'évolution des cultures de Charbon. Ann. Inst. Pasteur, 45, 1.

Burnet, F. M., Keogh, E. V. \& Lush, D. (1937). The immunological reactions of the filtrable viruses. Austr. J. exp. Biol. Med. Sci. 15, 227.

Cowles, P. B. (1931). The recovery of bacteriophage from filtrates derived from heated spore-suspensions. J. Bact. 22, 119.

Den Dooren de Jong, L. E. (1931). Studien über Bakteriophagie. I. Über Bac. megatherium und den darin anwesenden Bakteriophagen. Zbl. Bakt. (1. Abt. Orig.), 120, 1.

Foster, J. W. \& Perry, J. J. (1954). Intracellular events occurring during endotrophic sporulation in Bacillus mycoides. J. Bact. 67, 295. 


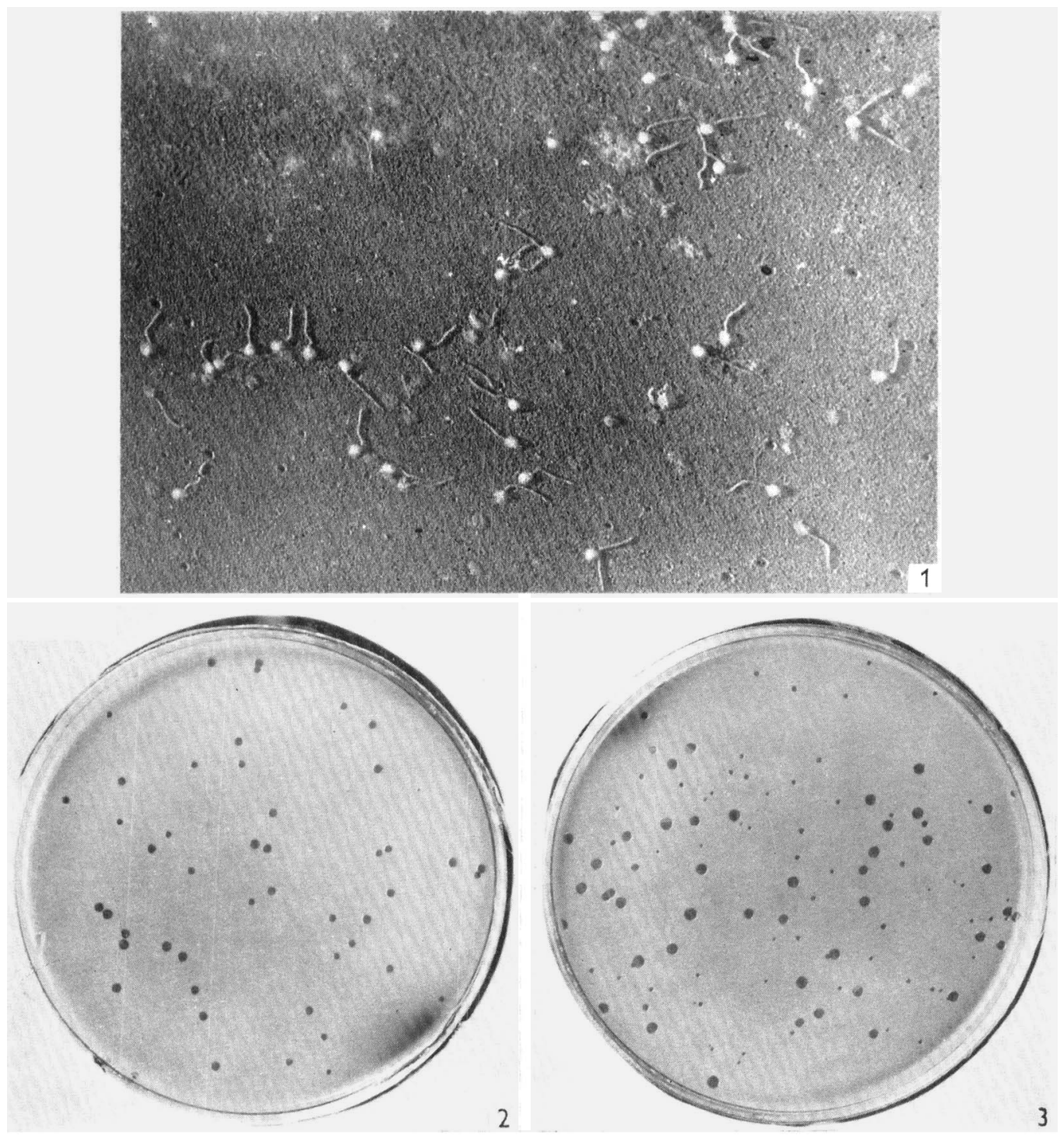


Journal of General Microbiology, Vol. 28, No. 1

Plate 2
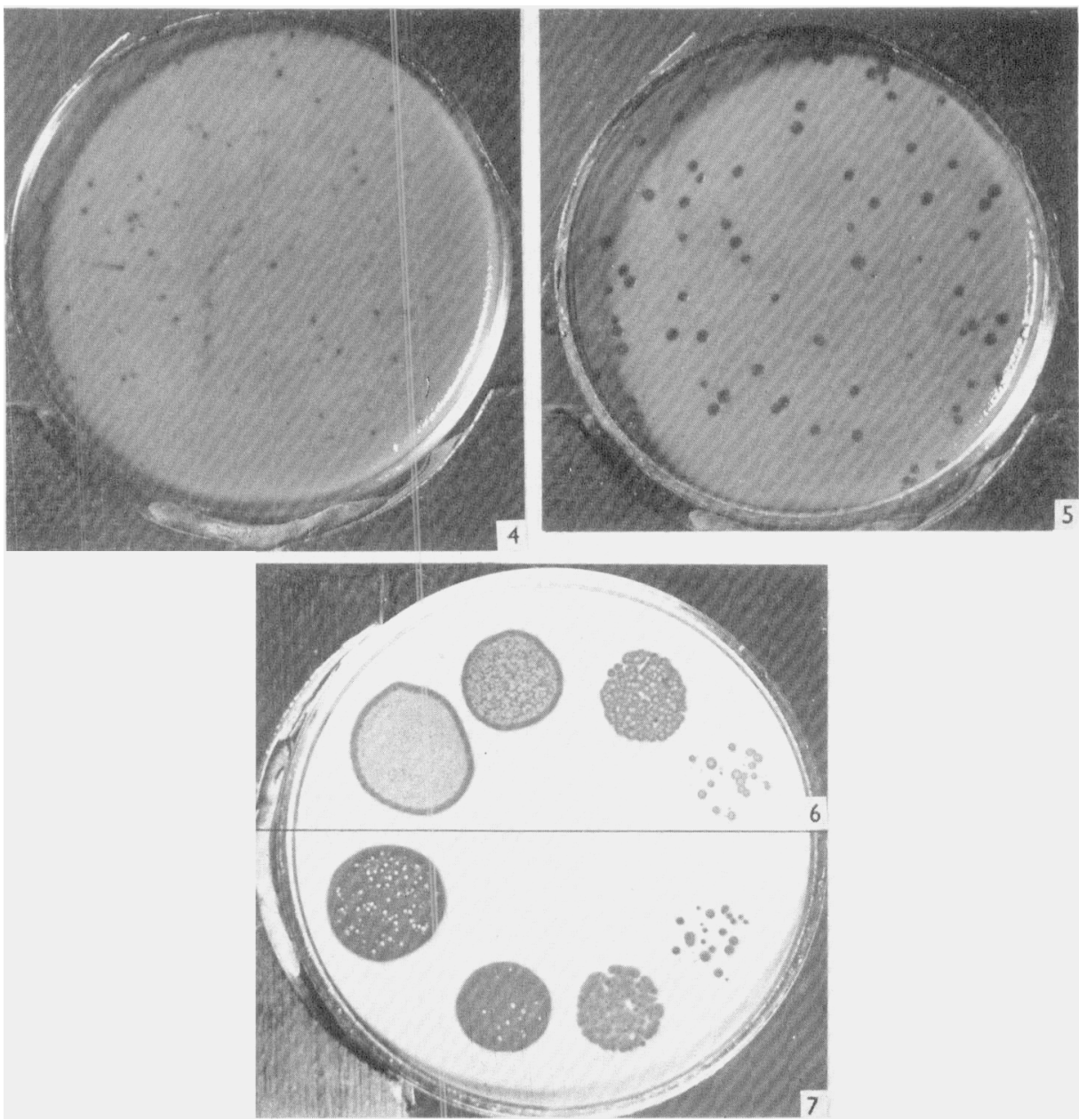

E. W. MEYNELL. 
Graziosi, F., Coppo, A. \& Donini, P. (1954). Indagine della struttura del fago BM mediante inattivazione con anticorpi specifici. Nuovi Ann. Ig. 5, 27.

Harjwick, W. A. \& Foster, J. W. (1952). On the nature of sporogenesis in some aerobic bacteria. J. gen. Physiol. 35, $90 \%$.

Ivanovics, G. (1962). 'The pathogenicity of Bacillus anthracis lysogenic with mutants of phage W. J. gen. Microbiol. 28, 87.

Jerne, N. K. \& Avegno, P. (1956). The development of the phage-inactivating properties of serum during the course of specific immunisation of an animal: reversible and irreversible inactivation. $J$. Immunol. 76, 200.

Kalmanson, G. M. \& Bronfenbrenner, J. (1943). Restoration of activity of neutralized biologic agents by removal of the antibody with papain. J. Immunol. 47, 387 .

Kellenberger, E. \& Arber, W. (1957). Electron microscopical studies of phage multiplication. 1. A method for quantitative analysis of particle suspensions. Virology, 3, 245.

McCloy, E. W. (1951). Studies on a lysogenic Bacillus strain. I. A bacteriophage specific for Bacillus anthracis. J. Hyg., Camb. 49, 114.

McCloy, E. W. (1953). Sporulation of bacteria despite infection by virulent phage. VI Congr. int. Microbiol. $2,210$.

MCCloy, E. W. (1958). Lysogenicity and immunity to Bacillus phage W. J. gen. Microbiol. 18, 198.

Nagano, Y. \& Oda, M. (1955). Adhésion du bactériophage neutralisé sur le bacille sensible. C.R. Soc. Biol., Paris, 149, 863.

Nungester, W. J. (1929). Dissociation of B. anthracis. J. infect. Dis. 44, 73.

Renaux, E. (1952). Culture de Bacillus anthracis en milieu calcique et en milieu oxalaté. Ann. Inst. Pasteur, 83, 38.

Sterne, M. (1937). Variation in Bacillus anthracis. Onderstepoort J. vet. Sci. 8, 271.

Wriss, E. (192\%). The bacteriophage-antibacteriophage reaction. J. Immunol. 13, 301.

Wollman, E. \& Wollman, E. (1936). Recherches sur le phénomène de Twort-d'Hérelle (Bactériophagie ou autolyse hérédo-contagieuse). Ann. Inst. Pasteur, 56, 137.

\section{EXPLANATION OF PLATES}

Plate 1

Fig. 1. Electronmicrograph of phage $\gamma^{\prime} ; \times 35,000$.

Fig. 2. A normal stock of phage $\alpha$ plated on Bacillus anthracis strain Davis; showing the unimodal distribution of plaque diameters.

Fig. 3. A stock of phage $\alpha$ plated on B. anthracis strain Davis after partial inactivation by serum, showing a mixture of normal and abnormally small plaques.

\section{Plate 2}

Fig. 4. Plaques formed on $B$. anthracis strain Davis $(\beta \mathrm{LC})$ by phage $\alpha \mathrm{S}$ grown on strain Davis.

Fig. 5. Plaques formed by phage $\alpha \mathbf{L}$ on $B$. anthracis strain Davis $(\beta \mathrm{LC})$.

Fig. 6. Serial dilutions of phage $\alpha$ plated on $B$. cereus strain $W$.

Fig. 7. Serial dilutions of phage $\alpha 2$ plated on $B$. cereus strain W. 